\title{
Designed Potent Multivalent Chemoattractants for Escherichia coli ${ }^{\text {¿ }}$
}

\author{
Jason E. Gestwicki, Laura E. Strong, ${ }^{\dagger}$ Sara L. Borchardt, Christopher W. Cairo, \\ Alexandra M. Schnoes and Laura L. Kiessling* \\ Departments of Chemistry and Biochemistry, University of Wisconsin-Madison, Madison, WI 53706, USA
}

Received 21 March 2001; accepted 2 May 2001

\begin{abstract}
Bacterial chemotactic responses are initiated when certain small molecules (i.e., carbohydrates, amino acids) interact with bacterial chemoreceptors. Although bacterial chemotaxis has been the subject of intense investigations, few have explored the influence of attractant structure on signal generation and chemotaxis. Previously, we found that polymers bearing multiple copies of galactose interact with the chemoreceptor Trg via the periplasmic binding protein glucose/galactose binding protein (GGBP). These synthetic multivalent ligands were potent agonists of Escherichia coli chemotaxis. Here, we report on the development of a second generation of multivalent attractants that possess increased chemotactic activities. Strikingly, the new ligands can alter bacterial behavior at concentrations 10 -fold lower than those required with the original displays; thus, they are some of the most potent synthetic chemoattractants known. The potency depends on the number of galactose moieties attached to the oligomer backbone and the length of the linker tethering these carbohydrates. Our investigations reveal the plasticity of GGBP; it can bind and mediate responses to several carbohydrates and carbohydrate derivatives. These attributes of GGBP may underlie the ability of bacteria to sense a variety of ligands with relatively few receptors. Our results provide insight into the design and development of compounds that can modulate bacterial chemotaxis and pathogenicity. (C) 2001 Elsevier Science Ltd. All rights reserved.
\end{abstract}

\section{Introduction}

Bacterial chemotaxis is an ideal response for probing the molecular mechanisms by which environmental stimuli influence cellular behavior. The signaling cascade is well-characterized, and there is a wealth of structural and functional knowledge available on the component proteins. ${ }^{1-5}$ Although the signal transduction pathway that results in chemotaxis is one of the best understood, how the molecular recognition of chemotactic agents influences amplification and propagation of signals remains unclear. Synthetic chemoattractants can elucidate the relationship between ligand recognition and chemotactic responses. Specifically, multivalent displays of the attractant galactose derived from ring-opening metathesis polymerization (ROMP) were recently found to be more potent bacterial chemoattractants than the corresponding monovalent ligands. ${ }^{6}$ Significantly, the

\footnotetext{
*Corresponding author. Tel.: +1-608-262-0541; fax: +1-608-2650764; e-mail: kiessling@chem.wisc.edu

${ }^{\dagger}$ Present address: Quintessence Biosciences, University Research Park, 505 S. Rosa Rd., Madison, WI 53719, USA.

Dedicated to Professor Peter B. Dervan on the occasion of his award of the Tetrahedron Prize.
}

valencies of these ligands influenced their activities: those with higher valency were more effective agonists. These results demonstrate that ligands of different valencies can be used to alter bacterial behavior, and they support a model ${ }^{7-9}$ of chemotactic signal amplification that involves receptor clusters. ${ }^{10,11}$ To illuminate the interplay between carbohydrate recognition and its influence on cellular behavior, we used molecular modeling methodology to design a second generation of multivalent chemoattractants.

The periplasmic binding protein GGBP recognizes the monosaccharides D-galactose, D-glucose, methyl- $\beta$-Dgalactoside, methyl- $\beta$-D-glucoside, L-arabinose, and Dxylose and mediates chemotaxis to and transport of these sugars. ${ }^{12-14}$ Upon interaction with its saccharide ligands, GGBP undergoes a conformational change to facilitate the interaction of bound GGBP to the membrane-anchored chemoreceptor Trg. Occupied Trg influences the distribution of phosphorylated signaling proteins, and this alteration results in a chemotactic response. ${ }^{1-4}$ The range of saccharides that GGBP recognizes suggests it possesses an accommodating binding site. Still, not all glucose and galactose derivatives can bind. For example, 3-O-methyl-D-glucoside is 
a poor chemoattractant. ${ }^{12}$ Given the tolerance of GGBP to substituents at the anomeric position of galactose, we reasoned that galactose could be presented from an oligomeric backbone through an anomeric substituent. On the basis of this analysis, we synthesized and tested chemoattractants that displayed galactose residues appended through an anomeric linker in our previous studies. ${ }^{6}$

The synthetic galactose derivatives, all of which possessed an anomeric substituent, were effective chemoattractants, although the monomer was somewhat less effective than unmodified galactose. Interestingly, molecular modeling studies suggested that an anomeric substituent should have a much more deleterious effect than that observed. A model of the galactose-bound GGBP structure determined by X-ray crystallography indicates that the anomeric hydroxyl group is oriented toward the bottom of the cleft of GGBP (Fig. 1A, C and E). ${ }^{15}$ In this location, modifications of the anomeric position should not be tolerated. Consequently, we set out to investigate how the carbohydrate was interacting with GGBP and to develop more effective ligands. Here, we report the design of galactose derivatives devised to interact more effectively with GGBP, the synthesis of the galactose-displaying targets, and the abilities of these monovalent and multivalent ligands to elicit chemotactic responses in Escherichia coli. The ligand parameters that we varied and tested include valency of galactose residues and the length of the anomeric linker. Our results provide insight into the structural features that influence the activities of natural and non-natural chemoattractants.

\section{Results}

The chemotactic activity that we observed previously for galactose derivatives was difficult to reconcile with the solid-state structure of galactose-bound GGBP. Consequently, we used molecular modeling to ascertain whether other orientations of the carbohydrate in the binding site would be permitted.

The program AutoDock was used to perform docking calculations of galactose binding to GGBP. ${ }^{16}$ One low energy orientation of galactose that positions the anomeric hydroxyl towards the solvent exposed entrance of the binding cleft was repeatedly observed by cluster analysis (Fig. 1B, D, and F). In this binding mode, the hydrogen bonds from the protein to three of the four hydroxyl groups were maintained (Fig. 1D) as were the van der Waals contacts to Trp 183. The root mean square distance (RMSD) between the alternative orientation and that observed in the solid-state structure was $1.54 \AA$. Using this model, we sought to optimize the structure of the anomeric linker connecting galactose to the oligomer backbone.

Inspection of the structure of GGBP indicated that an anomeric linker composed of 6-10 atoms would allow for optimal access of galactose to the binding cleft. A series of galactose derivatives containing oligoethyleneglycol spacers were modeled into the binding site using MacroModel, and of these, a triethyleneglycol linker appeared to be most favorable. Molecular dynamics simulations with this galactose derivative suggested that this substituent would be of sufficient length to minimize steric crowding (Fig. 1F). Thus, we set out to synthesize and test the chemotactic activity of the compounds predicted by modeling to function as more effective chemoattractants (Fig. 2).

To compare the activities of oligomers possessing different spacings between the galactose recognition element and the oligomeric backbone, we took advantage of the post-polymerization modification (PPM) strategy we had developed for ROMP. ${ }^{17}$ In the PPM approach, recognition elements are introduced after polymerization. Consequently, oligomers possessing alternative linkers can readily be synthesized and their biological activities compared. When the ruthenium initiator $\left[(\mathrm{Cy})_{3} \mathrm{P}\right]_{2} \mathrm{Cl}_{2} \mathrm{Ru}=\mathrm{CHPh}$ is used, the polymerization can be living. Moreover, the rate of initiation can exceed that of propagation with this complex; thus, oligomers of different valencies can be generated by varying the ratio of catalyst to monomer. ${ }^{18}$ Based on the most active chemoattractants we synthesized previously, $\mathrm{N}$ hydroxysuccinimidyl ester-containing polymers were prepared by ROMP with monomer to initiator ratios of $10: 1$ and $25: 1 .^{6}$ The $N$-hydroxysuccinimidyl esters undergo subsequent conjugation of recognition elements containing primary amines. ${ }^{17}$ The anomeric substituent was introduced by glycosylation of peracylated galactose with azido alcohol derived from triethyleneglycol. Removal of the acetate groups followed by reduction of the azide, generated the desired galactose moiety 4. Oligomers containing $N$-hydroxysuccinimide esters were prepared by ROMP using different monomer to initiator ratios $(10: 1$ and 25:1). PPM of these materials using amine $\mathbf{4}$ afforded galactosedisplaying oligomers 6 and 7. Conjugation of amine $\mathbf{4}$ to bicyclo[2.2.1]hept-5-ene-exo-2-carboxylic acid afforded monomer 5 (Fig. 3).

Molecular modeling studies predict that the ligands with the longer linker (5-7) would have a greater affinity for GGBP than the first generation ligands (1-3). To test this prediction, GGBP was purified from the periplasm of E. coli. ${ }^{19}$ Relative GGBP binding affinities were determined for the monomers with the short and long linkers by monitoring the increase in tryptophan fluorescence. A change in fluorescence accompanies ligand binding, presumably because ligands perturb the side chain microenvironment. ${ }^{20}$ By this measure, monomer 5 bound significantly tighter than its counterpart 1 . The relative dissociation constant, $K_{\text {rel }}$, for 1 was $110 \mathrm{nM}$ but that of 5 was 100 -fold lower, $1.1 \mathrm{nM}$. Thus, our strategy for ligand optimization was successful in affording ligands that bind more effectively; however, it is the ternary complex of liganded GGBP and the chemoreceptor Trg that elicits chemotaxis. Consequently, we sought to determine the effects of increased linker length on bacterial chemotactic responses.

Compounds 1-3 and 5-7 were introduced to $E$. coli at various concentrations by capillary ${ }^{21}$ and the 
concentration that resulted in a maximum chemotactic response was determined (Table 1). For all oligomers, the tabulated concentrations reflect the total galactose residue concentration not the concentration of oligomer. Compounds $\mathbf{6}$ and $\mathbf{7}$ exhibited improved activity over the corresponding first generation compounds (2 and 3). The activity of 6 was improved 4-fold over that of 2. Compound 7, which was prepared with a monomer to initiator ratio of $25: 1$, is the most active galactose-based chemoattractant known. In the assay used here, this multivalent ligand has potency that is 2.5 -fold greater than that of 3 and 10-fold greater than that of

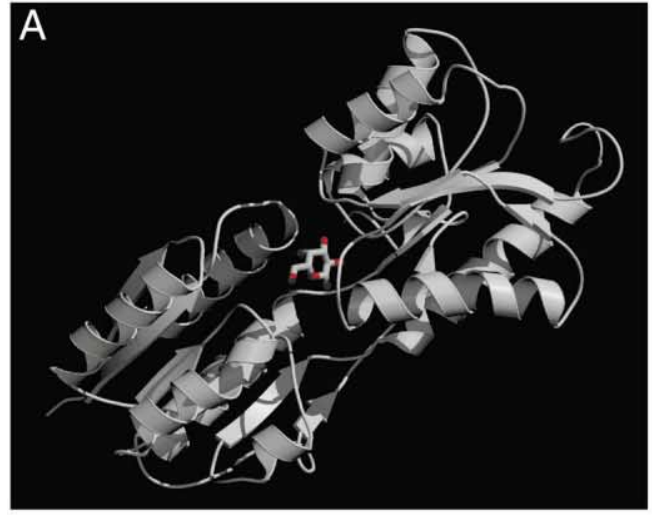

C
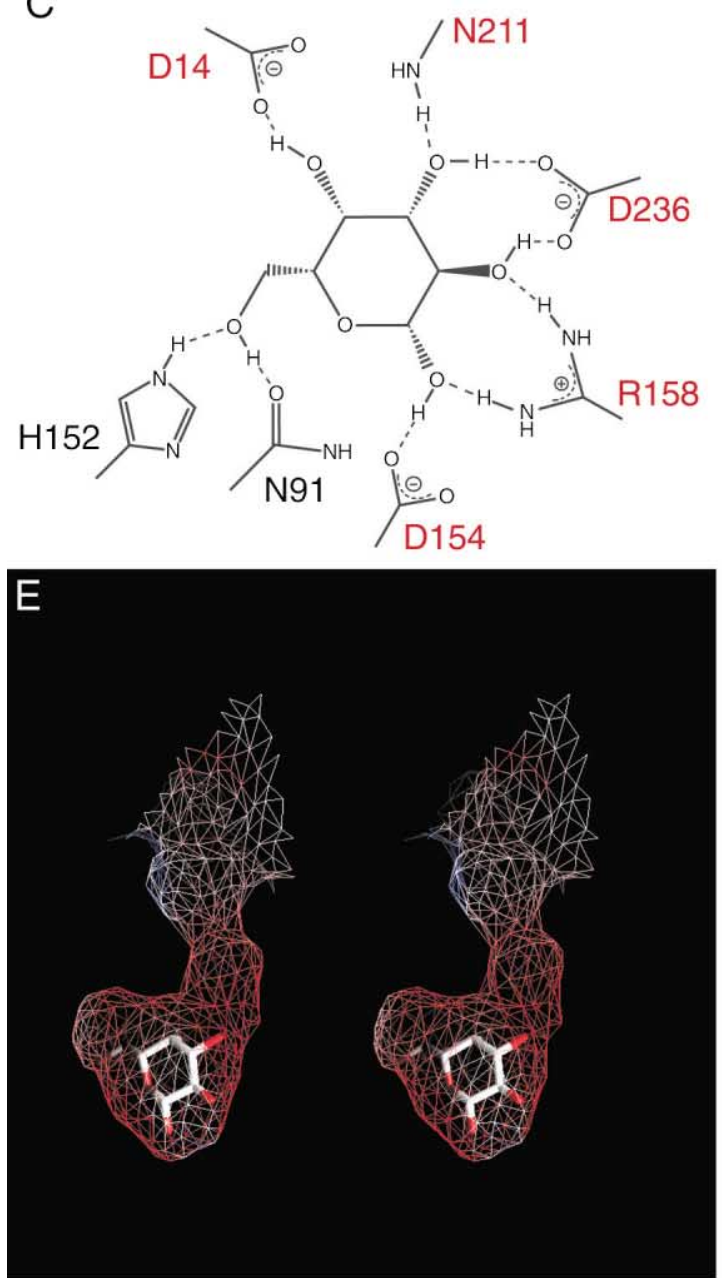

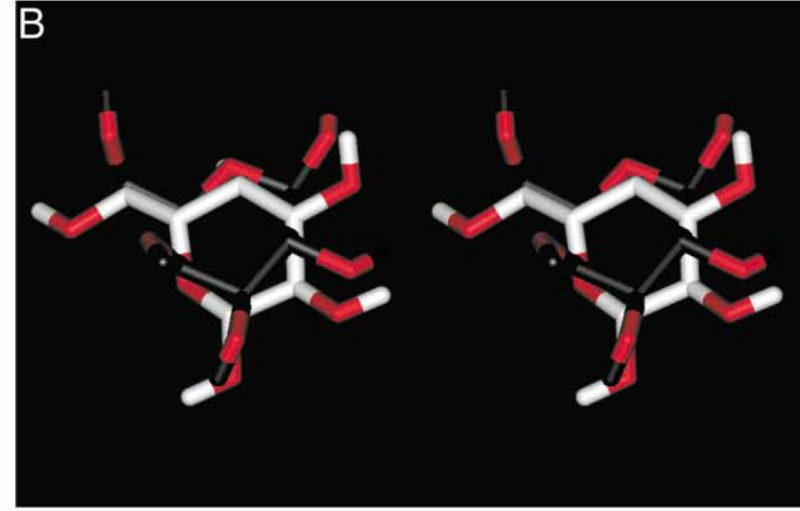

$\mathrm{D}$
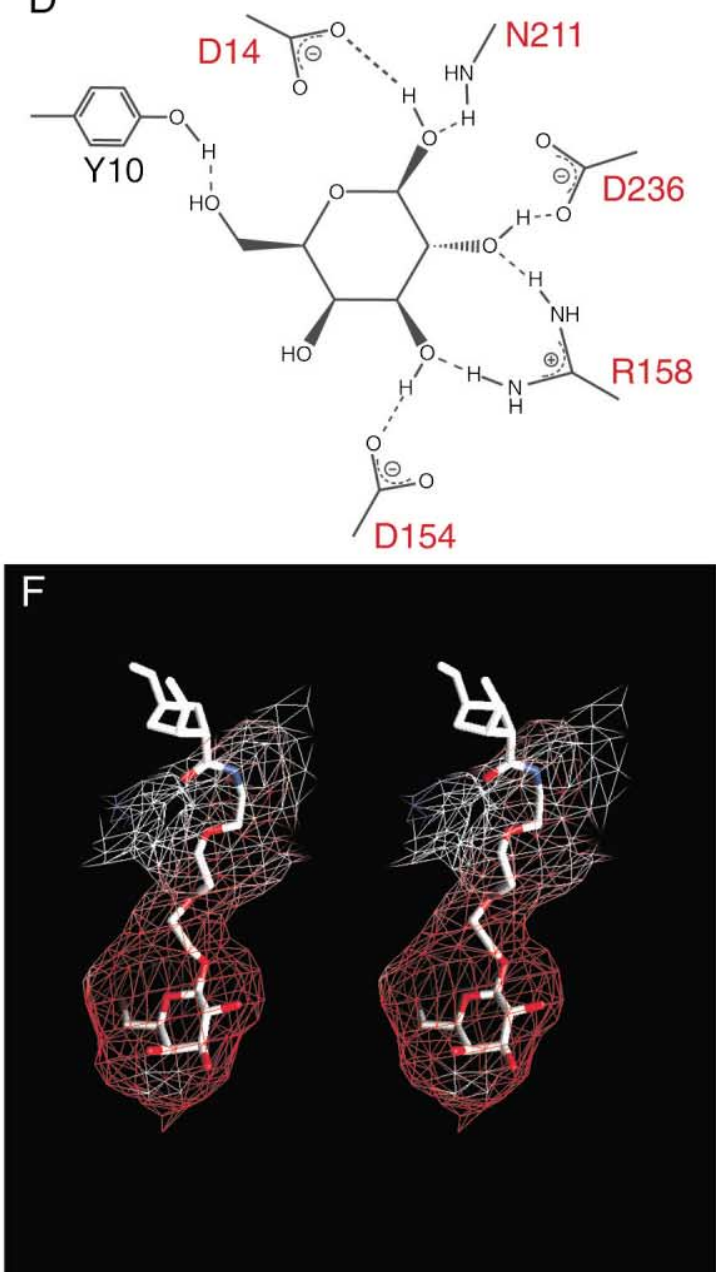

Figure 1. Structures of liganded GGBP and orientations of bound galactose. (A) Depiction of the structure of galactose-bound GGBP highlighting the position of the binding cleft between the two halves of the protein. ${ }^{15}$ The orientation of the binding cleft from this structure is approximately maintained in the remainder of the panels. The image was produced in Raster 3-D. ${ }^{31,32}$ (B) Stereoview of the orientation of galactose proposed from the structure as determined by X-ray crystallography (light gray) superimposed over the new orientation predicted by modeling (dark gray). Stereoimage was produced in WebLab Viewer Lite. (C) Depiction of the major hydrogen bonds to galactose proposed from the structure determined by X-ray crystallography. (D) Depiction of the major hydrogen bonds predicted from the alternative binding mode for galactose derivatives. (E) Stereoview surface diagram of the deep binding cleft, shown for the galactose-bound structure as determined by X-ray crystallography. (F) Binding cleft, shown with a bound monomer unit incorporating a triethyleneglycol linker. The surface images were produced in GRASP. 

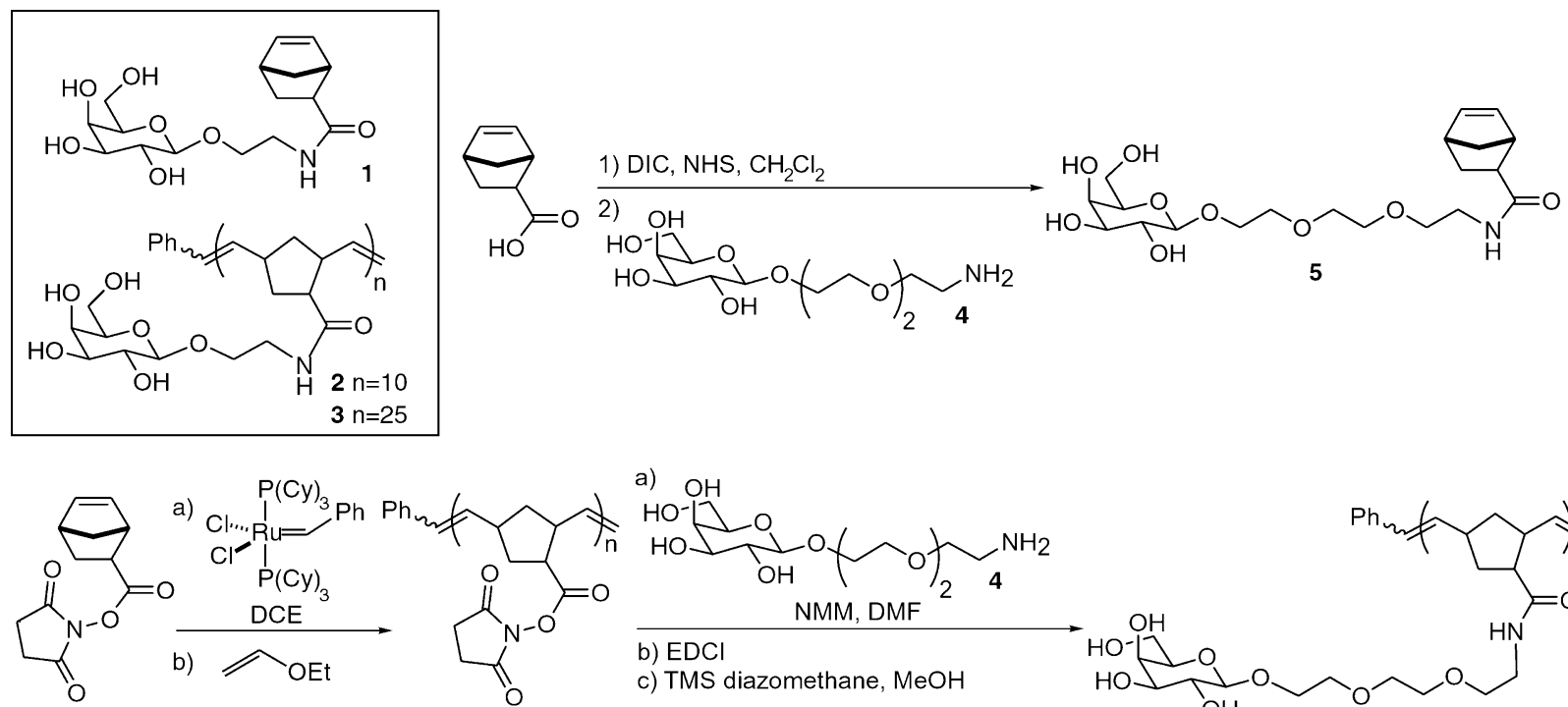<smiles>CCCCC1CC(C(=O)ON2C(=O)CCC2=O)C(C(C)C)C1</smiles>

a) $\mathrm{OH}$
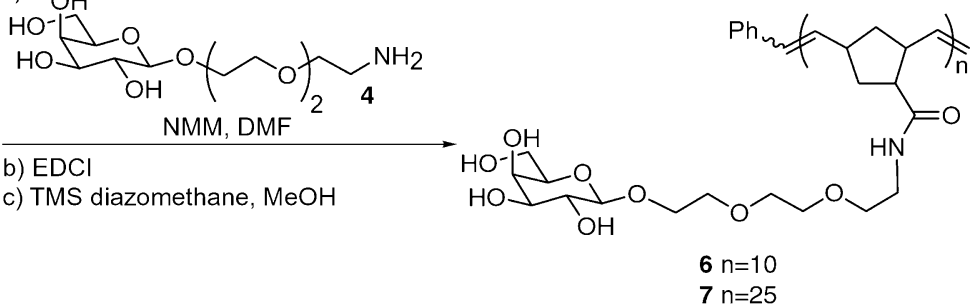

Figure 2. Chemical structures of synthetic chemoattractants and schemes for the synthesis of second-generation chemoattractants (5-7).

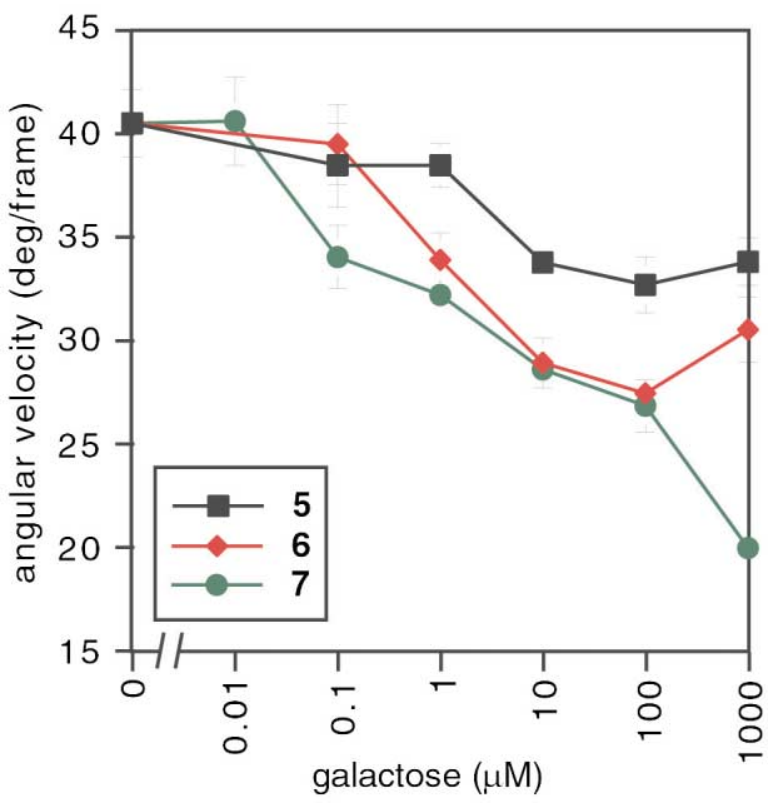

Figure 3. Dependence of bacterial behavioral responses on concentration and valency as measured by motion analysis. Values are the average angular velocity over the first $15 \mathrm{~s}$ after addition of attractant. Results are the average of 2-4 experiments performed in triplicate. Ligand concentration is based on total galactose concentration. Error bars represent the per second standard deviation and some are smaller than the symbols.

galactose. Alternatively, the activity calculated by the concentration of chemoattractant molecules indicates that 7 is approximately 250 -fold more active than galactose.

Following the determination of chemotactic activity, we then sought to directly examine the effects of the second generation chemoattractants on the behavior of $E$. coli by motion analysis. Motion analysis involves reconstruction of bacterial paths from digitized video frames for quantitation of behavior. ${ }^{22,23}$ This assay monitors chemokinetic rather than chemotactic activity as no gradient of attractant is established. It has the advan-
Table 1. Chemotactic activity

\begin{tabular}{lcc}
\hline Compd & Maximum chemotaxis $(\mathrm{mM})^{\mathrm{a}}$ & Relative potency \\
\hline Galactose & 1.0 & 1 \\
$\mathbf{1}$ & 1.0 & 1 \\
$\mathbf{2}$ & 1.0 & 1 \\
$\mathbf{3}$ & 0.25 & 4 \\
$\mathbf{5}$ & n.d. & 4 \\
$\mathbf{6}$ & 0.25 & 10 \\
$\mathbf{7}$ & 0.10 &
\end{tabular}

n.d., not determined.

aThe concentration of maximum chemotaxis is defined as the ligand concentration at which the largest number of bacteria are collected in the capillary assay. 
tage that the behavior of individual cells can be followed rather than the bulk response of a population, and it is widely used to determine the response of bacteria to attractants and repellants. ${ }^{6}$ Cellular response can be evaluated by angular velocity determinations. When a cell is tumbling frequently, the angular velocity is high; but when a chemoattractant is introduced, the angular velocity decreases as the bacterial path straightens. Thus, angular velocity is inversely proportional to the potency of the ligand; angular velocity decreases as activity increases. The change in the angular velocity of $E$. coli in response to 5-7 was dependent on both concentration and valency (Fig. 3). Multivalent ligand 7 exhibited the highest potencies at both high and low concentrations. Thus, as we observed previously with compounds 1-3, the valency of a ligand influences chemotactic potency. ${ }^{6}$ These results demonstrate the utility of this assay in determining relative bacterial responses to synthetic ligands.

To compare the potencies of all the first- and secondgeneration chemoattractants, we evaluated their threshold concentrations. Threshold concentration, as determined by motion analysis, is defined as the lowest concentration at which the angular velocity is significantly lower than the buffer-treated control. The galactose ligands bearing longer connecting units are active at lower threshold concentrations (Table 2). The potencies of multivalent ligands 6 and 7 are each approximately 10 -fold higher than the corresponding compounds $\mathbf{2}$ and 3. Consistent with our other observations, 7 is the most active ligand. Galactose derivative 7 possesses activity at a threshold concentration 10-fold lower than that of any of the other attractants; it is 100 -fold more potent than galactose. When activity is determined based on the number of attractant molecules, 7 is approximately 2500 -fold more potent than galactose. Thus, compound $\mathbf{7}$ is the most potent galactosederived chemoattractant reported.

\section{Discussion}

Although the signaling pathway that leads to bacterial chemotaxis has long served as a model for signal transduction systems, many mechanistic questions remain. One such issue is how bacteria can respond with high sensitivity to changes in chemoattractant concentration (e.g., ca. 10\%) over such a broad concentration range (i.e., 5 orders of magnitude). ${ }^{24}$ Moreover, an understanding of how stimulus binding affinity influences cellular behavior has yet to emerge. To explore these issues, we

Table 2. Results of motion analysis

\begin{tabular}{lcc}
\hline Compd & Threshold $(\mu \mathrm{M})$ & Relative potency \\
\hline Galactose & 10 & 1 \\
$\mathbf{1}$ & 10 & 1 \\
$\mathbf{2}$ & 10 & 1 \\
$\mathbf{3}$ & 1 & 10 \\
$\mathbf{5}$ & 10 & 1 \\
$\mathbf{6}$ & 1 & 10 \\
$\mathbf{7}$ & 0.1 & 100 \\
\hline
\end{tabular}

have generated synthetic compounds designed to serve as chemoattractants. Our objective is to illuminate the relationship between stimulus structure and its ability to influence cellular behavior. This information could result in new antimicrobial agents, as bacterial chemotaxis can be involved in bacterial pathogenicity. ${ }^{25,26}$

The multivalent ligands were synthesized to explore a mechanism for signal amplification that involves chemoreceptor communication through clustered arrays. ${ }^{7-9}$ In this model, ligand binding to one receptor can influence others in the cluster. Although attractive, there is little direct experimental evidence in support of such a mechanism. If receptor arrays are important, we postulated that ligands that can interact simultaneously with multiple receptors would thereby stabilize clusters and enhance communication between receptor oligomers. Thus, multivalent galactose derivatives can assemble multiple copies of GGBP; the resulting scaffolded assemblies of GGBP would more effectively promote clustering and activation of the chemoreceptors than their monovalent counterparts. Our data indicate the ligands such as 3 and $\mathbf{7}$, which can assemble multiple copies of GGBP, are more potent than ligands that are incapable (monovalent derivatives $\mathbf{1}$ and 5) or less capable (multivalent ligands 2 and 6) of binding multiple copies of protein. Multivalent galactose derivatives have been shown to cluster chemoreceptors in cells. ${ }^{6}$ The increased chemotactic ability of compound 7 relative to 3 may stem from the ability of 7, with its galactose residues suspended through a longer flexible linker, to more favorably cluster the chemoreceptors thereby eliciting more effective signal transduction.

By varying the length of the galactose tether, the relationship between GGBP binding constant and chemotactic activity could be assessed. We found that the ability of our galactose derivatives with anomeric substituents to bind GGBP can be enhanced at least 100fold by increasing the linker length. Interestingly, however, the difference in GGBP affinity between monovalent $\mathbf{1}$ and $\mathbf{5}$ was not manifested in their chemokinetic activities. Thus, optimizing a ligand for GGBP binding does not necessarily lead to an increase in its chemoattractant potency. Still, multivalent ligands with longer tethers separating galactose from the oligomeric backbone did exhibit improved chemotactic activity. For example, compound $\mathbf{6}$ is 4 -fold more active than $\mathbf{2}$ and the longer oligomer $\mathbf{7}$ is 2.5 -fold more potent than $\mathbf{3}$ in chemotaxis assays. Similarly, the responsive threshold concentrations for $\mathbf{6}$ and $\mathbf{7}$ are 10-fold lower than those for their counterparts $\mathbf{2}$ and $\mathbf{3}$, respectively. These results do not preclude a role for GGBP affinity in the promotion of chemotactic responses, but they do suggest that other factors are important. The observation that only the multivalent ligands exhibited an increase in potency is consistent with the aforementioned mechanism invoking signal amplification through chemoreceptor clustering.

The activities of the synthetic galactose derivatives provide an indication of the plasticity of $E$. coli sensing. The intermediary GGBP has a narrow and deep binding 
cleft, yet a variety of galactose derivatives are tolerated. Surprisingly, the protein has the ability to recognize and mediate a response to galactose derivatives with anomeric substituents despite the obvious steric clashes expected from the structure of the GGBP-galactose complex. These data highlight the versatility of GGBP in facilitating responses to sugars. The ability of GGBP to act as a mediator for a variety of sugars may be an evolutionary advantage: it allows bacteria to recognize many possible carbon sources. Despite having only four major chemoreceptors and six chemotaxis-mediating periplasmic binding proteins, ${ }^{27,28} \mathrm{E}$. coli is capable of chemotaxis in response to a remarkably large number of small molecule ligands and environmental conditions $\left(\mathrm{pH}\right.$, temperature, salt). ${ }^{12,29,30}$ The plasticity we have observed in the GGBP binding site may be advantageous for the development of synthetic ligands that influence chemotaxis and inhibit bacterial pathogenecity.

\section{Conclusions}

Bacterial chemotaxis is one of the most well understood biological responses. Many questions still remain about how chemotactic signal transduction is regulated and what molecular features of a ligand contribute to potent chemotactic activity. We have demonstrated that the potency of multivalent galactose-based chemo-attractants is high and that it can be enhanced significantly by increasing galactose epitope valency. Although the solid state structure of the GGBP-galactose complex suggests that galactose derivatives with anomeric substituents will not bind, bacteria respond to these derivatives through GGBP recognition. Our chemotactic data from monovalent and multivalent galactose derivatives suggest a mechanism for amplification of chemotactic signals. Specifically, ligand binding to clustered arrays of receptors results in propagation of the signal. We anticipate that additional variations in ligand architecture will further illuminate the mechanism(s) of agonist activity and lead to compounds capable of attenuating bacterial pathogenicity.

\section{Experimental}

\section{General methods}

${ }^{1} \mathrm{H}$ NMR spectra were recorded on a Bruker AC-300 $(300 \mathrm{MHz})$ spectrometer and chemical shifts are reported as ppm referenced to tetramethylsilane.

\section{Molecular modelling}

Surfaces of the binding site of GGBP were generated in GRASP 1.2 using coordinates retrieved from the protein data bank (PDB ID: 1GLG and 1GCG ). Monosaccharides were docked to GGBP (PDB ID: 1GLG) using AutoDock 2.4. For these runs the hydroxyl groups of the saccharides were allowed to rotate freely. Grid files for the docking simulations were $60 \AA$ on each side. The resulting conformations were analyzed by clusters. Pseudosymmetric transformations of galactose
(C1 to $\mathrm{C} 3, \mathrm{C} 4$ to $\mathrm{O} 5, \mathrm{O} 1$ to $\mathrm{C} 3$, etc.) were used to calculate an RMSD of $1.54 \AA$. Optimal lengths for ethylene glycol linkers were determined by introducing monomer units into the binding site of GGBP (1GLG with original ligand removed) using MacroModel 6.5 (Schrödinger, Inc., Portland, OR, USA). Each monomer was subjected to substructure molecular dynamics for 200 ps using the AMBER* force field and GBSA water solvation. Each run was then analyzed by observing the motion of the atoms in each linker unit to determine the optimal length. Figure 1A was produced in Raster 3-D v2.3 31,32 and $1 \mathrm{~B}$ was produced in WebLab Viewer Lite v4.0 (MSI, San Diego, CA, USA).

Synthesis of galactose-substituted polymers 6 and 7 . The synthesis of 2-\{2-[2-(2-amino-ethoxy)-ethoxy]-ethoxy\}$\beta$-D-galactopyranoside $4^{33}$ and bicyclo[2.2.1] hept-5-eneexo-2-carboxylic acid $N$-hydroxysuccinimide ester and the polymerization of bicyclo[2.2.1]hept-5-ene-exo-2carboxylic acid $N$-hydroxysuccinimide ester followed literature descriptions. ${ }^{17}$ The approximate ligand valency $(n)$ represents the ratio of monomer to catalyst used in the polymerization. The synthesis of 1-3 was described previously. ${ }^{6,34}$ Coupling of amine 4 to the product of the polymerization of bicyclo[2.2.1]hept-5ene-exo-2-carboxylic acid $N$-hydroxysuccinimide ester was performed as follows: Compound 4 (20.4 mg, $0.0788 \mathrm{mmol}), N$-methylmorpholine $(7.7 \mu \mathrm{L}, 0.070 \mathrm{mmol})$ and polymeric $N$-hydroxysuccinimide ester $(15.2 \mathrm{mg}$, $0.0647 \mathrm{mmol}$ ) were stirred in dimethylformamide for $24 \mathrm{~h}$. Diisopropylcarbodiimide $(11 \mu \mathrm{L}, 0.064 \mathrm{mmol})$ was added and stirring continued overnight. The dimethylformamide was removed under reduced pressure, and the resulting solid was washed three times with $1-2 \mathrm{~mL}$ of dichloromethane and ethanol. The solid was dried, and (trimethylsilyl)diazomethane $(35 \mu \mathrm{L}, 0.070 \mathrm{mmol})$ and methanol $(350 \mu \mathrm{L})$ were added, and the reaction was stirred overnight. The reaction was quenched upon addition of water, and the solvent was removed under reduced pressure. The solid was dissolved in $\mathrm{H}_{2} \mathrm{O}$ and dialyzed extensively against $\mathrm{H}_{2} \mathrm{O}$ to remove impurities from the coupling reaction. The solution was filtered through a 0.25 micron filter and the solvent was removed under reduced pressure to give a tan solid (15.4 mg, 71\%). ${ }^{1} \mathrm{H}$ NMR (300 MHz $\left.\mathrm{D}_{2} \mathrm{O}\right) \delta 7.3$ (br m, $0.278 \mathrm{H}), 5.5-4.9(\mathrm{br}, 2 \mathrm{H}), 4.0-3.0(\mathrm{br} \mathrm{m}, 14 \mathrm{H}), 2.5-$ 2.15 (br m, 2 H), 1.9-1.4 (br, 2 H), 1.1-0.9 (br, 2 H).

Expression and purification of GGBP. GGBP was purified from the periplasm of the E. coli strain HB929 (a generous gift of G. Hazelbauer, Washington State-Pullman) using the shockate method. ${ }^{19}$ Contaminating monosaccharides were removed by denaturation of GGBP with $2.0 \mathrm{M} \mathrm{GdHCl}$ and subsequent extensive dialysis at $4{ }^{\circ} \mathrm{C}$. The protein was $>90 \%$ pure as judged by SDS-PAGE and present primarily in the unliganded form after dialysis. ${ }^{35}$ Protein concentration was determined by Bradford assay using bovine serum albumin as a standard.

Determination of GGBP-ligand dissociation constants. GGBP was resuspended in $10 \mathrm{mM}$ HEPES buffer $\mathrm{pH}$ 7.2 at a final concentration of $16.7 \mu \mathrm{g} / \mu \mathrm{L} .{ }^{20}$ Galactose 
or a galactose derivative ( $\mathbf{1}$ or $\mathbf{5}$ ) was suspended in 10 mM HEPES pH 7.2 at appropriate concentrations. A solution of the galactose derivative $(10 \mu \mathrm{L})$ was added to a solution of GGBP $(90 \mu \mathrm{L})$. The solution was vigorously mixed and then allowed to equilibrate for at least $30 \mathrm{~min}$ at $22^{\circ} \mathrm{C}$. The total volume $(100 \mu \mathrm{L})$ was then added to a quartz cuvette and an excitation scan was performed (emission $330 \mathrm{~nm}, 5 \mathrm{~nm}$ slit widths). The excitation intensity at $280 \mathrm{~nm}$ was recorded and used to determine dissociation constants. Experiments were performed on a Hitachi F-4500 spectrofluorimeter at $22{ }^{\circ} \mathrm{C}$. Relative dissociation constants $\left(K_{\text {rel }}\right)$ were determined from the data sets by fitting the titration curves to the equation: ${ }^{36}$

$F=\left(F_{\max } \times[\right.$ galactose $\left.]\right) /\left([\right.$ galactose $\left.]+K_{\text {rel }}\right)$

where $\Delta F$ is the $\%$ change in fluorescence intensity at $280 \mathrm{~nm}, \Delta F_{\max }$ is the maximum \% change in fluorescence, and [galactose] is the total concentration of saccharide. Curve fitting was performed using DeltaGraph 4.5. Experiments were performed in quadruplicate and error was approximately $4 \%$.

Capillary accumulation assay. E. coli strain AW405 from an overnight culture were grown in LB (Luria Bertani broth) to $\mathrm{OD}_{550}$ 0.4-0.6, washed twice with $10 \mathrm{mM}$ phosphate buffer and utilized in the capillary accumulation assay as previously described. ${ }^{6,21}$ All concentrations shown represent the total galactose concentration. Experiments are the average of at least three trials performed in duplicate and error is approximately $20 \%$.

Motion analysis. E. coli AW405 from an overnight culture were grown in $\mathrm{LB}$ with $1 \mathrm{mM}$ galactose to an $\mathrm{OD}_{550}$ of $0.4-0.6$ and then washed twice with $10 \mathrm{mM}$ phosphate buffer. Motion analysis was performed by computational analysis of digitized video microscopy results as previously described. ${ }^{6,23}$ No adaptation was detectable during the first $15 \mathrm{~s}$ of the response.

\section{Acknowledgements}

The authors thank G. Hazelbauer (Washington State University) for providing GGBP expression strain HB929 and J. Adler (UW-Madison) for supplying $E$. coli strain AW405. This research was supported in part by the NIH (GM55984). J.E.G. thanks the Biotechnology Training Program for a predoctoral fellowship (GM08349). L.E.S. was supported by an NIH predoctoral fellowship (GM18750). The NMR facility in the Department of Chemistry (UW-Madison) is funded by grants from the NSF (CHE-9208463) and NIH (RR08389).

\section{References and Notes}

1. Stock, J. B.; Lukat, G. S.; Stock, A. M. Annu. Rev. Biophys. Biophys. Chem. 1991, 20, 109.

2. Hazelbauer, G. L.; Berg, H. C.; Matsumura, P. Cell 1993, $73,15$.

3. Falke, J. J.; Bass, R. B.; Butler, S. L.; Chervitz, S. A.; Danielson, M. A. Annu. Rev. Cell Dev. Biol. 1997, 13, 457.

4. Bren, A.; Eisenbach, M. J. Bacteriol. 2000, 182, 6865.

5. Shimizu, T. S.; Le Novére, N.; Levin, M. D.; Beavil, A. J.; Sutton, B. J.; Bray, D. Nature Cell Biol. 2000, 2, 792.

6. Gestwicki, J. E.; Strong, L. E.; Kiessling, L. L. Chem. Biol. 2000, 7, 583 .

7. Levit, M. N.; Liu, Y.; Stock, J. B. Mol. Microbiol 1998, 30, 459 .

8. Bray, D.; Levin, M. D.; Morton-Firth, C. J. Nature 1998 , 393,85

9. Duke, T. A. J.; Bray, D. Proc. Natl. Acad. Sci. U.S.A. 1999, 96, 10104.

10. Maki, N.; Gestwicki, J. E.; Lake, E. M.; Kiessling, L. L.; Adler, J. J. Bacteriol. 2000, 182, 4337.

11. Maddock, J. R.; Shapiro, L. Science 1993, 259, 1717.

12. Adler, J.; Hazelbauer, G. L.; Dahl, M. M. J. Bacteriol. 1973, 115, 824.

13. Hazelbauer, G. L.; Adler, J. Nature New Biol. 1971, $230,101$.

14. Boos, W. Eur J. Biochem. 1969, 10, 66.

15. Vyas, M. N.; Vyas, N. K.; Quiocho, F. A. Biochemistry 1994, 33, 4762.

16. Morris, G. M.; Goodsell, D. S.; Huey, R.; Olson, A. J. Comput. Aided Mol. Design 1996, 10, 293.

17. Strong, L. E.; Kiessling, L. L. J. Am. Chem. Soc. 1999, 121, 6193.

18. Fraser, C.; Grubbs, R. H. Macromolecules 1995, 28, 7248.

19. Anraku, Y. J. Biol. Chem. 1968, 243, 3116.

20. Boos, W.; Gordon, A. S.; Hall, R. E.; Price, H. D. J. Biol. Chem. 1972, 247, 917.

21. Adler, J. J. Gen. Microbiol. 1973, 74, 77.

22. Berg, H. C.; Brown, D. A. Nature 1972, 239, 500.

23. Sager, B. M.; Sekelsky, J. J.; Matsumura, P.; Adler, J. Anal. Biochem. 1988, 173, 271.

24. Mesibov, R.; Ordal, G. W.; Adler, J. J. Gen. Physiol. 1973, 62, 203.

25. Foynes, S.; Dorrell, N.; Ward, S. J.; Stabler, R. A.; McColm, A. A.; Rycroft, A. N.; Wren, B. W. Infect. Immun. 2000, 68, 2016.

26. Cangelosi, G. A.; Ankenbauer, R. G.; Nester, E. W. Proc. Natl. Acad. Sci. U.S.A. 1990, 87, 6708.

27. Grebe, T. W.; Stock, J. Curr. Biol. 1998, 8, R154.

28. Quiocho, F. A.; Ledvina, P. S. Mol. Microbiol. 1996, 20, 17.

29. Mesibov, R.; Adler, J. J. Bacteriol. 1972, 112, 315.

30. Taylor, B. L.; Zhulin, I. B.; Johnson, M. S. Annu. Rev. Microbiol. 1999, 53, 103.

31. Kraulis, P. J. J. Appl. Crystallogr. 1991, 24, 946.

32. Bacon, D.; Anderson, W. F. J. Mol. Graphics 1988, 6, 219.

33. Chernyak, A. Y.; Sharma, G. V. M.; Kononov, L. O.; Krishna, P. R.; Levinsky, A. B.; Kochetkov, N. K.; Rao, A. V. R. Carbohyd. Res. 1992, 223, 303.

34. Manning, D. D.; Strong, L. E.; Hu, X.; Beck, P. J.; Kiessling, L. L. Tetrahedron 1997, 53, 11937.

35. Rasched, I.; Shuman, H.; Boos, W. Eur. J. Biochem. 1976, 69, 545.

36. Martin, G. E. M.; Rutherford, N. G.; Henderson, P. J. F.; Walmsley, A. R. Biochem. J. 1995, 308, 261. 